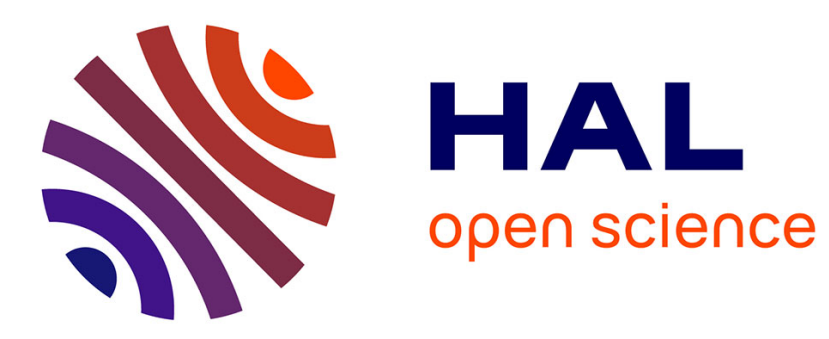

\title{
Redesign of the MMOC microgripper piezoactuator using a new topological optimization method.
}

Mathieur Grossard, Christine Rotinat-Libersa, Nicolas Chaillet

\section{To cite this version:}

Mathieur Grossard, Christine Rotinat-Libersa, Nicolas Chaillet. Redesign of the MMOC microgripper piezoactuator using a new topological optimization method.. IEEE/ASME International Conference on Advanced Intelligent Mechatronics, AIM'2007., Sep 2007, ETH Zürich, Switzerland. sur CD ROM - 6 p. hal-00171674

\author{
HAL Id: hal-00171674 \\ https://hal.science/hal-00171674
}

Submitted on 12 Sep 2007

HAL is a multi-disciplinary open access archive for the deposit and dissemination of scientific research documents, whether they are published or not. The documents may come from teaching and research institutions in France or abroad, or from public or private research centers.
L'archive ouverte pluridisciplinaire HAL, est destinée au dépôt et à la diffusion de documents scientifiques de niveau recherche, publiés ou non, émanant des établissements d'enseignement et de recherche français ou étrangers, des laboratoires publics ou privés. 


\title{
Redesign of the MMOC microgripper piezoactuator using a new topological optimization method
}

\author{
M. Grossard, C. Rotinat-Libersa, N. Chaillet
}

\begin{abstract}
This paper presents a new method developed for the optimal design of piezoactive compliant mechanisms. It is based on a flexible building blocks method, called FlexIn, which uses an evolutionary approach, to optimize a truss-like structure made of passive and active piezoelectric building blocks. An electromechanical approach, based on a mixed finite element method, is used to establish the model of the piezoelectric blocks. A planar monolithic compliant microactuator is synthesized by the optimization method, based on the specifications drawn from a piezoelectric microgripper prototype (MMOC). Finally, some performances comparisons between the optimally FlexIn synthetized gripper and the previous gripping system demonstrate the interests of the proposed optimization method for the design of microactuators, microrobots, and more generally for adaptronic structures.
\end{abstract}

Index Terms - Actuator design, compliant mechanisms, microrobotics, genetic algorithm, piezoelectricity, microgripper, topology optimization.

\section{INTRODUCTION}

$I_{v}^{N}$ $\mathrm{N}$ microrobotic applications, pick-and-place tasks are widespread operations. Most of micromanipulators, mainly laboratories prototypes, use tightening fingers as end-effectors, which are intuitive tools for the user, and can address a significant part in the resolution of microhandling problems. Micromanipulation, that is to say the handling of objects with dimensions from $1 \mu \mathrm{m}$ to $1 \mathrm{~mm}$, concerns many domains: handling or characterisation of samples (e.g. biological cells) and assembly of micromechanical devices (e.g. microcomponents, microgears, etc). General overviews of microgrippers, and classifications according to the principle of actuation used (electrostatic force, thermal effect, magnetic principle, shape memory alloys, fluidic principle, piezoelectric ceramics), or to their dimensions, versatility and integration degrees can be found in [1] and [2].

Most often, these devices are compliant mechanisms, i.e. single-bodies, elastic continua flexible structures that transmit a motion by undergoing elastic deformation, as

Manuscript received January 15, 2007.

M. Grossard is with both the Laboratoire d'Automatique de Besançon (LAB CNRS / ENSMM / UFC), Besançon 25000, France, and CEA LIST, Interactive Robotics Unit, Fontenay-aux-Roses, F-92265 France (e-mail: mathieu.grossard@ens2m.fr).

C. Rotinat-Libersa is with the CEA LIST, Interactive Robotics Unit, Fontenay-aux-Roses, F-92265 France (e-mail: christine.rotinatlibersa@cea.fr).

N. Chaillet is with the Laboratoire d'Automatique de Besançon (LAB CNRS / ENSMM / UFC), Besançon 25000, France (e-mail: nicolas.chaillet@ens2m.fr). opposed to jointed rigid body motions of conventional articulated mechanisms. They are coupled with smart materials for actuation. Indeed, using compliant mechanisms for the design of small scale systems is of a great interest, because of simplified manufacturing, reduced assembly costs, reduced kinematic noise, no wear, no backlash, and ability to accommodate unconventional actuation schemes.

One type of smart material typically used to actuate microrobotic structures is PZT piezoceramic. Such active material allows designing light actuation devices, and offers the advantages of a high energy density and a high output force, when compared to conventional actuation principles at small scales [3]. When integrated inside a compliant mechanism, piezoelectric actuators can exert actuation forces to the host structure without any external support. They can also be manufactured into the desired shape, while making realistic the realization of piezoelectric monolithic compliant mechanisms, such as microgrippers [4].

To improve their behavioral performances, it can be useful to optimize such devices from the first designing step, taking into account versatile microrobotic criteria. But, few studies consider the optimal design of such "smart structures" [5]. Because of the restricted range of motion of piezoelectric materials (only about $0.1 \%$ strain), a number of papers address the problem of designing coupling structures to act as stroke amplifiers of the piezoelectric actuator [6], [7], [8]. Opposite to these methods, where the piezoelectric elements in the structure are predetermined, a large body of work related to optimization of active structures, deals with the optimal location of actuators on a given structure [9]. Another general approach to optimally design smart structures is to simultaneously [10] or separately [11] optimize the actuator size. Finally, only a few studies consider the optimization of the shape of monolithic PZT actuators [12].

A more global systematic design approach, where topology optimization of the structure is used, as well as that of integrated piezoelectric actuators (i.e. location and size), has been developed to design monolithic compliant piezoactive mechanisms [13]. This method is based on the flexible building blocks method, called FlexIn ("Flexible Innovation"), developed at the CEA LIST [14], [15].

This paper is organized as follows: firstly, we will briefly review the underlying idea of the FlexIn methodology for the design of smart compliant mechanisms, and the main steps leading to piezoelectric blocks models (section II). To demonstrate the interest of this new optimal synthesis method for the design of efficient microrobotic devices (section III), we consider the synthesis of a planar monolithic compliant micro-actuator device, from the 
specifications of a piezoelectric micromanipulator, called MMOC, developed at the LAB. Finally, their performances are compared and analysed to demonstrate the interests of the FlexIn optimization method, for the design of unconventional micro-actuators for microrobotic uses.

\section{FLEXIN: A COMPLIANT MECHANISMS STOCHASTIC DESIGN METHODOLOGY}

In this section, we briefly present the flexible building blocks method, which has been implemented for the optimal design of micromechanical planar mechanisms in a software called FlexIn (developed with MATLAB ${ }^{\circledR}$ ) [2], [14], [15], and its evolution for the use of piezoelectric materials [13]. It uses a multi-objective evolutionary algorithm approach for the optimal design of smart compliant mechanisms made of an assembly of elementary passive and active compliant building blocks, chosen in two specific libraries.

\section{A. Compliant building blocks}

Two libraries of compliant elements in limited number are proposed in FlexIn. These bases are composed respectively of 36 and 19 elements of passive and piezoactive blocks, made of beams assembly (figure 1). They are sufficient to build a high variety of topologies. In particular, the various topologies of piezoactive blocks allow them to furnish multiple coupled degrees of freedom, thus generating more complex movements with only one building block. Moreover, the block feasibility related to fabrication process constraints can also be taken into account at this stage.

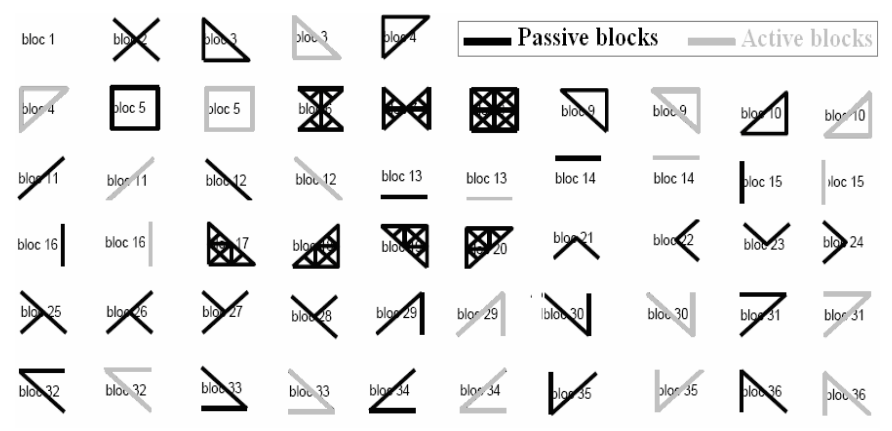

Figure 1. Passive (black) and active (grey) libraries of compliant building blocks, for planar compliant mechanisms synthesis using FlexIn.

\section{B. Principle of the method and design parameters}

The purpose of FlexIn is to optimally design realistic compliant structures. The specification of a planar compliant mechanism problem considers specific boundary conditions: fixed frame location, input (actuators), contacts and output (end-effector). Different types of actuation principles can be used: either external or internal force/displacement actuators defined at particular nodes of the mesh [2], or integrated piezoactive elements taken from the active library [13]. The design method consists in searching for an optimal distribution of allowed building blocks, as well as for the optimal set of structural parameters and materials. The location of fixed nodes and that of the actuators and/or piezoactive blocks can also be considered as optimisation parameters. The topology optimization method, inspired from [16], uses a genetic algorithm approach, which allows true multicriteria optimisation and the use of these discrete variables (figure 2). The algorithm is structured as follows:

- Discrete variable parameterization of compliant mechanisms considering conception requirements (mesh size, topology, material and thickness, boundary conditions),

- Evaluation of individuals (design criteria calculation),

- Stochastic operators for the optimization (modification of compliant mechanisms description).

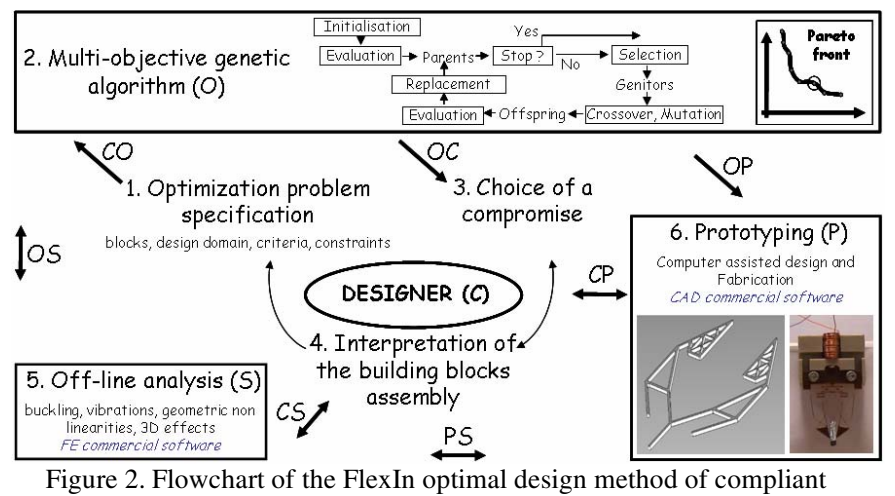
structures (multicriteria optimization).

\section{Multi criteria genetic algorithm}

Many fitness functions are available in FlexIn, thus allowing the optimal design of devices within a wide schedule of conditions: displacement and force at the output port, strain energy (SE), mutual strain energy (MSE), maximal stress (yield or fatigue strength), geometric advantage (GA), mechanical advantage (MA), mass, etc. Multi-degrees of freedom mechanisms design can also be considered. The optimization algorithm generates a set of pseudo-optimal solutions (see 2 in figure 2) in the case of multicriteria optimization problem (and obviously only one optimal solution for monocriterion optimization). The designer can choose, interpret and analyse the obtained structures that best suit his design problem (see 3 to 5 in

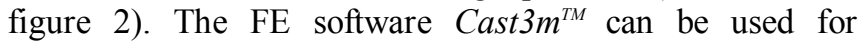
subsequent FEA, to analyse and validate the chosen design solution for criteria not considered during the optimization stage.

\section{Electro-mechanical FE model of the piezoactive blocks}

In FlexIn, it is assumed that the compliant mechanisms are undergoing structural deformations, mainly due to the bending of the beams constituting the blocks.

Thus, the models of the blocks are obtained considering Navier-Bernoulli beam type finite elements. Structural parameters of each rectangular block are height, width and thickness. Material characteristics of each block are parameterized by Young's modulus, Poisson's ratio, yield strength, density, and piezoelectric coefficients for the piezoactive blocks.

To allow the calculation of different criteria, FlexIn uses the FE model of each block of the libraries. To obtain the FE formulation of the piezoelectric blocks, a model of a piezoelectric beam is first needed. 


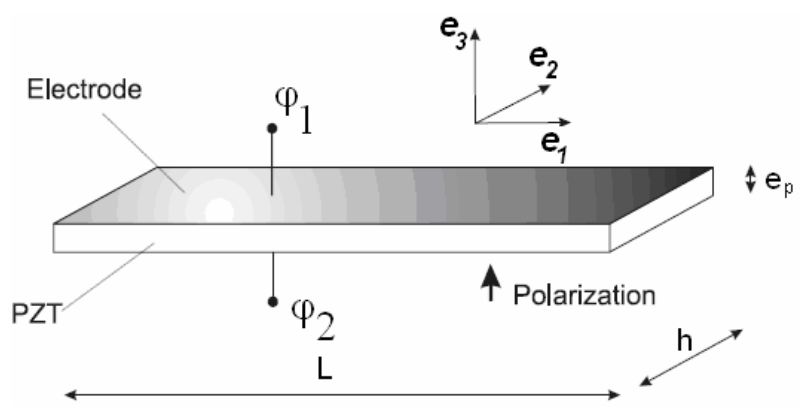

Figure 3. Thickness-polarized piezoelectric beam transducer with electroded surfaces, and orientation in the material reference frame $\left(\mathrm{e}_{1}, \mathrm{e}_{2}\right.$, $\left.\mathrm{e}_{3}\right)$.

We consider that the piezoceramic beams constituting the blocks are perfectly bonded to electrodes at their lower and upper faces (figure 3). Exploiting the transverse effect of piezoelectricity, longitudinal deformation $S_{11}$ along $L$ dimension is generated under the transverse electric field $E_{3}$. Considering the one-dimensional form of piezoelectricity equation along the length direction of the beam, the piezoelectric coupling matrix $d$ and the stress-free electric permittivity matrix $\varepsilon^{t}$ are each represented by a single coefficient, $d_{31}$ and $\varepsilon_{33}$ respectively, and the electric-free compliance matrix $s^{E}$ is represented by $s_{11}^{E}$. The subscript " $t$ " denotes the transpose of a matrix. Hence, within the piezoelectric beam, the constitutive relations for the strain $S_{11}$ and electric displacement $D_{3}$, as functions of stress $T_{11}$ and electric field $E_{3}$, take the form [17]:

$$
\left\{\begin{array}{l}
S_{11} \\
D_{3}
\end{array}\right\}=\left[\begin{array}{ll}
s_{11}^{E} & d_{31} \\
d_{31} & \varepsilon_{33}^{T}
\end{array}\right]\left\{\begin{array}{c}
T_{11} \\
E_{3}
\end{array}\right\} .
$$

The superscripts " $E$ " and " $T$ " refer to values taken respectively at constant electric and stress fields.

The displacement field over a planar beam element is described from its longitudinal $u$, tangential $v$ and rotational $\omega$ components at $x_{p}$ curvilinear abscissa (figure 4 ), and is related to the corresponding node values $\eta=\left(\begin{array}{lllll}u_{A} & v_{A} & \omega_{A} & u_{B} & v_{B}\end{array}\right.$ $\left.\omega_{B}\right)^{t}$ in the beam coordinate system $R_{p}=\left(A, x_{p}, y_{p}, z_{p}\right)$. From Hamilton's Principle modified for general electromechanical system [18], [19], the model of the active beam takes the following form:

$$
M \ddot{\eta}+D \dot{\eta}+K \eta=G \Phi+f_{r},
$$

where $M, D, K$ and $G$ are respectively the mass, damping, stiffness and electromechanical coupling beam matrices. $\Phi=\left[\begin{array}{ll}\varphi_{1} & \varphi_{2}\end{array}\right]^{t}$ is the vector representing the electric potentials on the upper and lower faces of the piezoelectric beam. Matrix $G$ in (2) induces piezoelectric loads, which makes the actuator beam expand or contract proportionally to the external controlled potential difference $\left(\varphi_{1}-\varphi_{2}\right)$. The forces vector $f_{r}$, is due to the variational mechanical work terms, and is written (figure 4):

$$
f_{r}=\left(\begin{array}{llllll}
R_{A}^{x} & R_{A}^{y} & H_{A}^{z} & R_{B}^{x} & R_{B}^{y} & H_{B}^{z}
\end{array}\right)_{R_{p}}^{t} .
$$

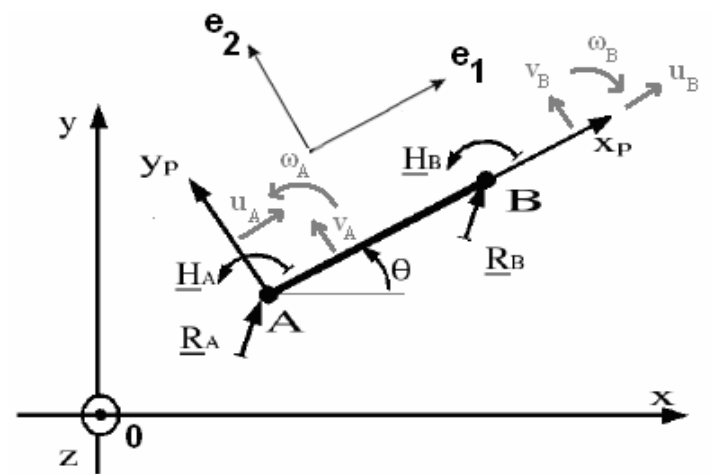

Figure 4. Curvilinear coordinates of the piezoelectric beam AB, and its orientation in the global coordinate system $(0, \mathrm{x}, \mathrm{y}, \mathrm{z})$. $\underline{\mathrm{R}}$ and $\underline{\mathrm{H}}$ represent the nodal force and moment at the beam extremities.

Some particular piezoelectric materials (e.g. the crystallographic class $6 \mathrm{~mm})$ are isotropic in $(0, \mathrm{x}, \mathrm{y})$ plane. Thus, mechanical and piezoelectric characteristics of a beam are invariant by in-plane rotation $\theta$ around z-axis (figure 4), so that equation (2) is the general 2D FE model for all the beams constituting a planar truss. The results obtained by our 2D active blocks FE model have been validated in static conditions by a commercial 3D multiphysics finite element software (under various boundary conditions) [13].

The stiffness, damping, and mass matrices of each block are then calculated numerically, considering every combination of the discrete values allowed for the structural optimization variables. Then, they are condensed to reduce the numerical problem size, which is of great interest when using a genetic algorithm approach for multi-objective optimal design. The calculation of the different reduced matrices of each valued-block is done one time only at the beginning of the optimal design problem (before running the genetic algorithm), thus saving running time. The global dynamic behaviour of a structure results from the mass, damping, stiffness and electromechanical coupling matrices assembly of the constitutive blocks, and is done at each step for each individual during the optimisation process.

In the following, because of the static criterion used, it will only be considered the static model with $K$ and $G$ matrices defined by :

$$
K=Y\left(\begin{array}{cccccc}
\frac{A}{L} & 0 & 0 & -\frac{A}{L} & 0 & 0 \\
& \frac{12 I}{L^{3}} & \frac{6 I}{L^{2}} & 0 & -\frac{12 I}{L^{3}} & \frac{6 I}{L^{2}} \\
\frac{4 I}{L} & 0 & -\frac{6 I}{L^{2}} & \frac{2 I}{L^{2}} \\
(s y m) & & \frac{A}{L} & 0 & 0 \\
G=Y h d_{31}\left(\begin{array}{cccccc}
1 & 0 & 0 & -1 & 0 & 0 \\
-1 & 0 & 0 & 1 & 0 & 0
\end{array}\right)_{R_{p}}^{t},
\end{array}\right.
$$

where $I$ designs the inertia moment of the cross section $A$ of the beam, and $Y$ its Young's modulus. The latter is: $Y=1 / s_{11}$. 


\section{E. Fitness evaluation: the output displacement example}

A static criterion specified in many schedules of conditions of mechatronic devices is the output displacement of the compliant mechanism. Here, we briefly present the way used to determine the output displacement of a monolithic piezoactive structure, as it is done in FlexIn.

The static behaviour of the structure is described by the blocks matrices assembly, and can be written as :

$$
K_{\text {global }} \eta_{\text {global }}=F_{\text {global }},
$$

where $K_{\text {global }}, \eta_{\text {global }}$ and $F_{\text {global }}$ are respectively the global stiffness matrix, the nodal displacements, and the total electromechanical loads, resulting from the both mechanical and piezoelectric contributions of the whole structure. Making distinction between imposed loads $F_{i}$ and imposed displacements $\eta_{d}$, (6) can also be rearranged into the following form :

$$
\left[\begin{array}{ll}
K_{i i} & K_{i d} \\
K_{d i} & K_{d d}
\end{array}\right]\left(\begin{array}{c}
\eta_{i} \\
\eta_{d}
\end{array}\right)=\left(\begin{array}{c}
F_{i} \\
F_{d}
\end{array}\right)
$$

Unknown displacements $\eta_{i}$ and, among them, the output displacement (fitness of interest here), is deduced by :

$$
\eta_{i}=K_{i i}^{-1}\left(F_{i}-K_{i d} \eta_{d}\right) .
$$

\section{OPTIMAL SYNTHESIS OF A PLANAR MONOLITHIC COMPLIANT PIEZOACTUATOR FOR MICROGRIPPERS}

\section{A. Piezoactuator specifications of MMOC microgripper}

The "Microprehensile Microrobot On Chip" (MMOC) is a compact microgripper (figure 5), developed at the Laboratoire d'Automatique de Besancon (LAB), composed of two main parts: the piezoactuator and the end-effector (figure 5) [20], [21].
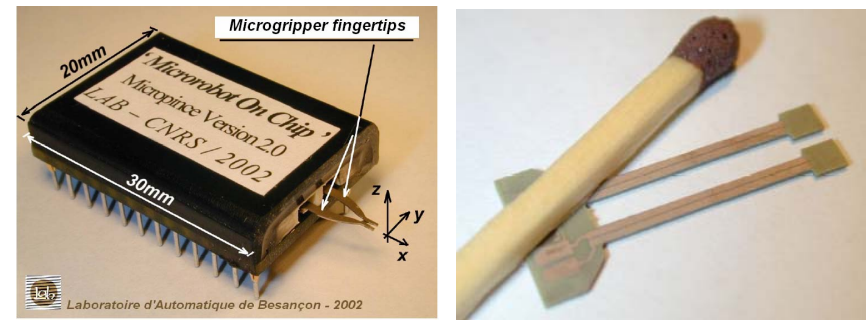

Figure 5. One version of a MMOC microgripper developped at LAB (left), and the piezoceramic actuator (right), on which the finger tips (seen on left) are mounted.

The piezoactuator consists of two parallel piezoceramic PZT PIC 151 bimorphs (figure 5), machined using an ultrasonic process. Each parallel bimorph is independent, and able to provide two uncoupled degrees of freedom. The piezoceramic bimorph contains two superimposed actuated $200 \mu \mathrm{m}$-thick layers, to produce independent movements along $\mathrm{y}$ and $\mathrm{z}$ directions.

As shown in figure 6, for (x-y) in-plane deformations, the voltages on two adjacent and two opposite electrodes are $V_{y}$ and $-V_{y}$. The directions of the electric fields $E_{1}$ and $E_{2}$, oriented along $\mathrm{z}$ axis, are opposite. Thus, the position of the upper layer of the actuator, located under the upper electrode at voltage $V_{y}$, is stretched along $x$ axis proportionally to the piezoelectric constant of the material $\mathrm{d}_{31}: \delta_{\mathrm{x}}=-\mathrm{d}_{31} \mathrm{~V}_{\mathrm{y}} / \mathrm{e}_{\mathrm{p}}$ (with $\mathrm{d}_{31}<0$ in general), where $\mathrm{e}_{\mathrm{p}}$ is the thickness of one piezoelectric layer. As the field under the adjacent electrode is the opposite, the portion of the plate under this electrode contracts. As the lower plate undergoes exactly the same deformations, the global deformation of the bimorph is a flexion in the $(\mathrm{x}, \mathrm{y})$ plane, resulting mainly in a displacement along $\mathrm{y}$ axis. The mechanical and electrical static characteristics of the MMOC actuator are given in the second column of table 1 .

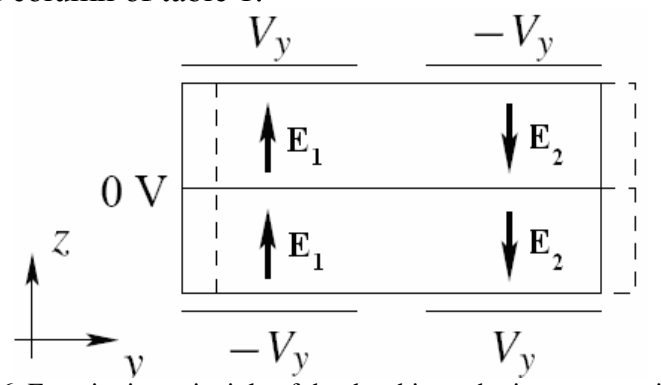

Figure 6. Functioning principle of the duo-bimorph piezoactuator in (x-y) plane. Structure at rest is presented with continuous lines; the deformed actuator with dashed ones.

\section{B. Optimization problem specifications}

The optimal synthesis method presented in section II has been used to redesign the in-plane piezoactuator part of MMOC, considering a symmetric monolithic compliant mechanism made of the same piezoelectric material [22].

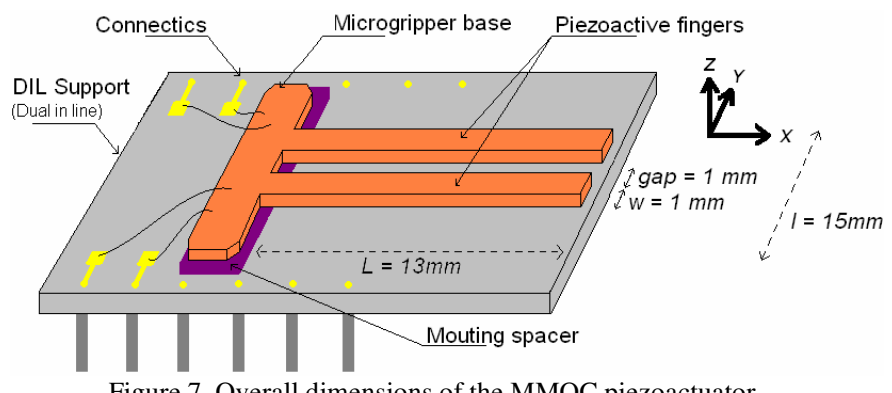

Figure 7. Overall dimensions of the MMOC piezoactuator.

To take advantage of the maximum size allowed for the actuator inside the MMOC system (figure 7), the half microactuator topology is considered to have a maximal size of $13 \mathrm{~mm} \times 7.5 \mathrm{~mm}$. It will be built with an assembly of both passive and active piezoelectric blocks to be optimized (figure $8)$. Actually, the active blocks are those which are bonded with electrodes, exploiting the piezoelectric actuator effect. For the optimal synthesis run, the number of active blocks in the half gripper can vary between one and nine. When external voltages are applied to the electrodes, the output node of the structure has to move horizontally and to produce a gripping force (figure 8). The potential difference for each active blocks can be chosen either $+100 \mathrm{~V}$ or $-100 \mathrm{~V}$ (table 1). The specifications of two optimisation problems, called A and $\mathrm{B}$, are detailed in figure 8 and Table 1. Each of these two optimisation problems consider more than $10^{28}$ possible candidate structures. The size ratio of the blocks can vary as $1 \leq b_{\max } / b_{\min } \leq 5$ and $1 \leq a_{\max } / a_{\min } \leq 8$ (figure 8). The thickness of the planar structure is taken constant and equal to $200 \mu \mathrm{m}$. Output free stroke and blocking force (i.e. gripping force in 
our application) are the objective functions to maximize simultaneously.

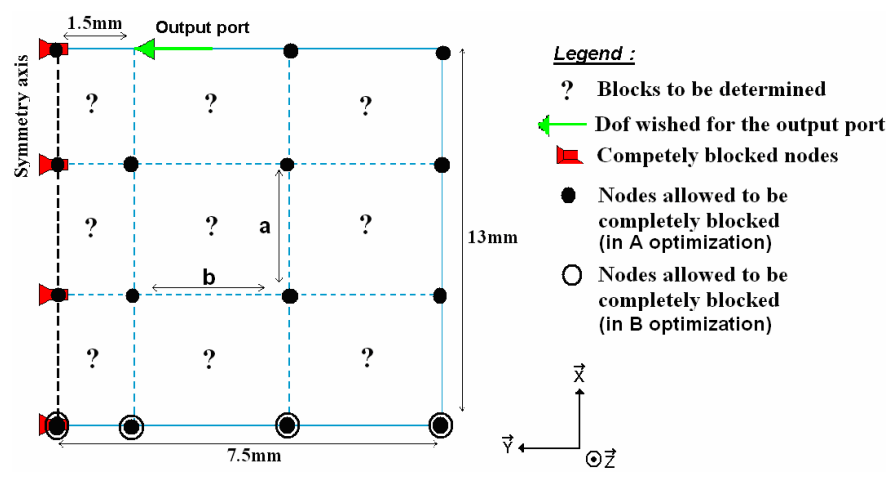

Figure 8. Half-mesh of the symmetric PZT compliant micro-actuator with imposed and permitted boundary conditions (vertical symmetric axis).

\section{Results}

The two optimization problems A and B were solved. The best compromises are kept, when the genetic algorithm does not find any new pseudo-optimum during 250 subsequent generations. Both A and B set of pseudo-optimal solutions can be represented on a Pareto front, giving their gripping force and output displacement performances (figure 9). The designer can choose among these solutions.

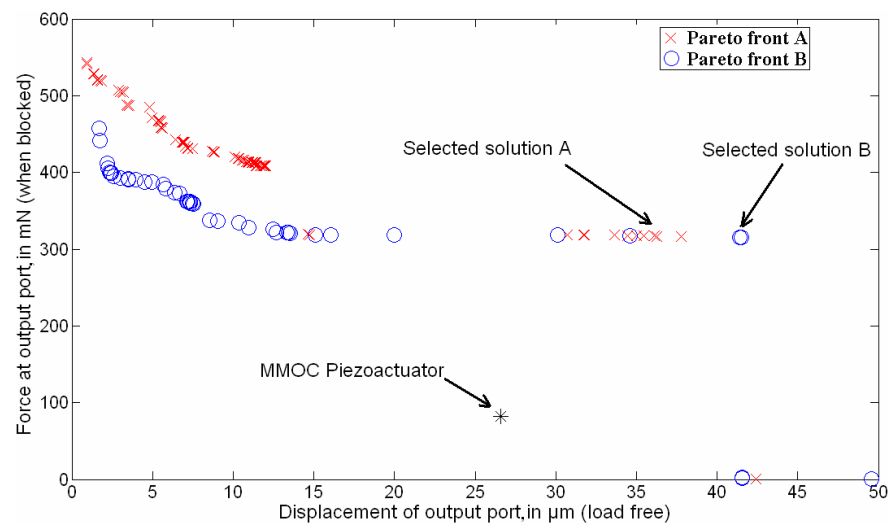

Figure 9. Pareto fronts A and B of compliant piezoactuators synthesized using FlexIn (genetic parameters used: population of 200 individuals, mutation probability of $40 \%$ on genes and individuals, stagnation criteria of 250 generations).

Both Pareto fronts show that the optimal synthesis method can generate piezoactuators designs, whose characteristics in terms of displacement, as well as in gripping forces are largely better than the bimorph structure of the MMOC piezoactuator. It shows the interest of the proposed method for the design of smart piezoactive structures with better performances than intuitive designed devices. Let us note that Pareto front $\mathrm{A}$ is globally above Pareto front B, except for high values of output displacement, for which pseudo-optimal microgrippers performances tend to be equivalent. For example, a selected pseudo-optimal solution is given in table 1 for each optimization. To illustrate the performances of the obtained grippers, figure 10 shows their deflection and gripping force versus the activation voltage, in comparison with the MMOC piezoactuator.

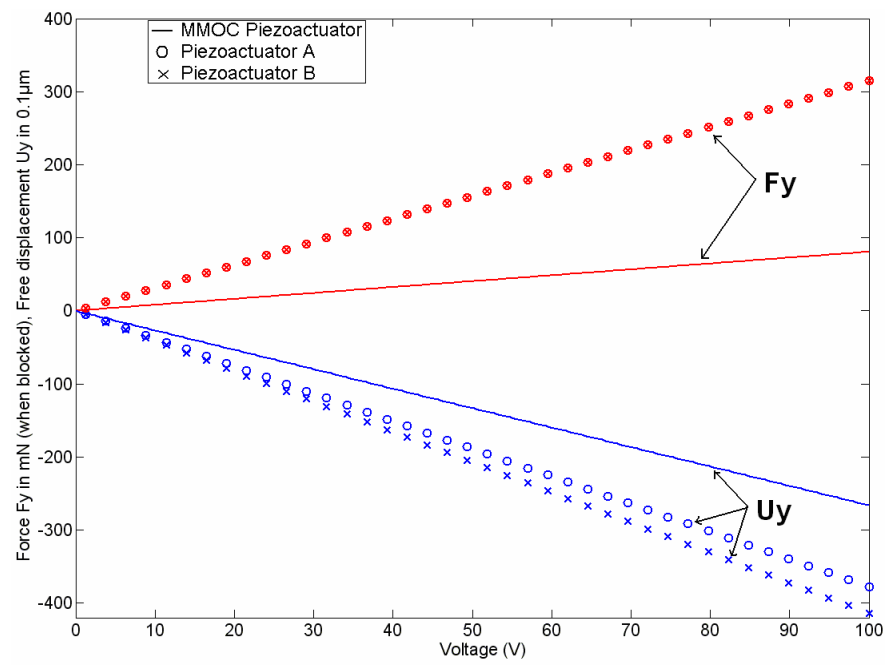

Figure10. Comparison of deflection and blocking force versus voltage gap for MMOC, A and B piezoactuators.

\section{CONCLUSION}

A new concept of optimal design method for smart compliant mechanisms has been presented. This method can consider a smart compliant mechanism as an assembly of passive and active compliant building blocks made of PZT, so that actuators are really integrated in the structure. The use of blocks allows a discrete variable parameterization of the design problem, which help reducing the search space. The example of the optimal design of a gripper actuator has proved that the method can furnish innovating and very efficient solutions, with better performances than well-known actuation schemes such as unimorph or bimorph PZT actuators, widespread in the design of microrobotic manipulators. Thus, complex multi-objective design problems can be solved by FlexIn to take advantage, for example, of the whole space available to generate high performances microgrippers prototypes.

Other optimality criteria are currently developed by the authors, considering not only the mechanical behaviour, but also the controllability and the observability of the structure output port. Another perspective is to take advantage of the direct piezoelectric effect, to consider as well force sensor integration inside monolithic structures. Microgrippers prototypes resulting from an optimal design with FlexIn will be realized soon for micromanipulation tasks.

\section{ACKNOWLEDGMENT}

The authors would like to thank J. Agnus from the Laboratoire d'Automatique de Besançon, who provided the characteristics of the MMOC system. 


\begin{tabular}{|c|c|c|c|}
\hline \multirow{2}{*}{ Characteristics } & \multirow{2}{*}{$\begin{array}{c}\text { MMOC } \\
\text { Piezoactuator }\end{array}$} & \multicolumn{2}{|c|}{ Selected optimal compliant microactuators } \\
\hline & & $\mathbf{A}$ & B \\
\hline \multicolumn{4}{|c|}{ Optimization problem specifications } \\
\hline Voltage gap between electrodes & $100 \mathrm{~V}$ & $100 \mathrm{~V}$ & $100 \mathrm{~V}$ \\
\hline Optimized variables & $\begin{array}{c}\text { No } \\
\text { optimization } \\
\text { (intuitive } \\
\text { design) }\end{array}$ & $\begin{array}{ll}\text { - } & \text { Active and passive } \\
\text { topologies } \\
\text { - } & \text { Size ratio of the blocks } \\
\text { - } & \text { Fixed nodes positions } \\
\text { (cf. figure } 8 \text { ) } \\
\text { - } \\
\text { Sign of the voltage gap } \\
\text { (i.e. }+100 \mathrm{~V} \text { or }-100 \mathrm{~V} \text { ) }\end{array}$ & $\begin{array}{ll}\text { - } & \text { Active and passive } \\
\text { topologies } \\
\text { - } & \text { Size ratio of the blocks } \\
\text { - } & \text { Fixed nodes positions } \\
\text { (cf. figure } 8 \text { ) } \\
\text { - } \\
\text { Sign of the voltage gap } \\
\text { (i.e. }+100 \mathrm{~V} \text { or }-100 \mathrm{~V})\end{array}$ \\
\hline \multicolumn{4}{|l|}{ Results } \\
\hline Maximal Y-stroke & $26.6 \mu \mathrm{m}$ & $37.8 \mu \mathrm{m}$ & $41.4 \mu \mathrm{m}$ \\
\hline Maximal Y-gripping force & $81.8 \mathrm{mN}$ & $316.8 \mathrm{mN}$ & $315.9 \mathrm{mN}$ \\
\hline $\begin{array}{c}\text { Gripper half-topology model } \\
\text { under FlexIn }\end{array}$ & $\begin{array}{l}\text { (see figure } 5 \\
\text { and } 7 \text { ) }\end{array}$ & & \\
\hline
\end{tabular}

Table 1. Specifications of the optimization problems and performances of the symmetric A and B piezoatuators, in comparison with the MMOC piezoactuator (bold lines refer to piezo-actuated blocks, whereas fine lines refer to piezo-passive ones)

\section{REFERENCES}

[1] Agnus J., Nectoux P., Chaillet N., "Overview of microgrippers and micromanipulation station based on a MMOC microgripper", Proceedings of the IEEE International Symposium on Computational Intelligence in Robotics and Automation, CIRA, Espoo, Finland, 2005, pp. 117-123.

[2] Rotinat-Libersa C., Perrot Y., Friconneau J.-P., "Potentialities of optimal design methods and associated numerical tools for the development of new micro- and nano- intelligent systems based on structural compliance - An example -", IARP- IEEE/RAS-EURON JointWorkshop on Micro and Nano Robotics, Paris, France, 2006, 11 p.

[3] Chaillet N., Bourjault A, in La microrobotique, Ed. Hermès Sciences Publications, 2002, pp. 165-197.

[4] Breguet J.M., and al., "Monolithic piezoceramic flexible structure for micromanipulation", $9^{\text {th }}$ International Precision Engineering Seminar and $4^{\text {th }}$ International Conference on Ultraprecision in Manufacturing Engineering, Braunschweig, Germany, 1997, pp. 397-400.

[5] Frecker M., "Recent advances in optimization of smart structures and actuators", Journal of Intelligent Material Systems and Structures, Vol.14, April/May, 2003, pp. 207-216.

[6] Frecker M., Canfield S., "Optimal design and experimental validation of compliant mechanical amplifiers for piezoceramic stack actuators", Journal of Intelligent Material Systems and Structures, Vol. 11, 2000, pp. 360-369.

[7] Kota S., "Tailoring unconventional actuators using compliant transmissions: design methods and applications", IEEE/ASME Transactions on Mechatronics, Vol. 4, December 1999, pp. 396-408.

[8] Lau G.K., and al., "Systematic design of displacement -Amplifying mechanisms for piezoelectric stacked actuators using topology optimization", Journal of Intelligent Material Systems and Structures, Vol. 3985, 2000, pp. 583-591.

[9] Barboni R., and al., "Optimal placement of PZT actuators for the control of beam dynamics", Smart Material and Structures, 2000, pp $110-120$

[10] Maddisetty H., Frecker M., "Dynamic topology optimization of compliant mechanisms and piezoceramic actuators", ASME Journal of Mechanical Design, Vol. 126, Iss. 6, 2002, pp. 975-983.
[11] Abdalla M., and al., "Design of a piezoelectric actuator and compliant mechanism combination for maximum energy efficiency", Smart Material and Structures, Vol. 14, 2005, pp. 1421-1430.

[12] Nelli Silva E.C., Kikuchi N., "Design of piezoelectric transducers using topology optimization", Smart Material and Structures, USA, Vol. 8, 1999, pp. 350 -365.

[13] Grossard M., and al., "Flexible building blocks method for the optimal design of compliant mechanisms using piezoelectric material", $12^{\text {th }}$ IFToMM World Congress, France, 2007 (in press).

[14] Bernardoni P., and al., "A new compliant mechanism design methodology based on flexible building blocks", Smart Material and Structures, USA, Vol. 5383, 2004, pp. 244-254.

[15] Bernardoni P., "Outils et méthodes de conception de structures mécaniques à déformations réparties et actionnement discret applications en microrobotique", PhD Thesis realized at the CEA, University Paris 6, France, 2004.

[16] Deb K., and al., "A fast elitist non-dominated sorting genetic algothm for multi-objective optimization: Nsga-II", Proc. of the 6th Int. Conf. on Parallel Problem Solving from Nature, France, 2000, pp. 849-858.

[17] ANSI/IEEE Std. 176-1987 IEEE Standard on piezoelectricity, 1987.

[18] Hagood N. W., Chung W. H., Von Flotow A., "Modelling of piezoelectric actuator dynamics for active structural control", Journal of Intelligent Material Systems and Structures, Vol. 1, 1990, pp. 327354.

[19] Piefort V., "Finite element modelling of piezoelectric active structures", PhD Thesis, Faculty of Applied Sciences, Bruxelles, Belgium, 2001.

[20] R. Perez, and al., "Modeling, fabrication, and validation of a highperformance 2-dof piezoactuator for micromanipulation", IEEE/ASME Transactions on Mechatronics, Vol. 10, No. 2, April 2002, pp. 161-171.

[21] De Lit P., Agnus J., Chaillet N., "Analytical modeling and finite element analysis of a monolithic piezoelectric actuator offering two uncoupled degrees of freedom", Proceedings of the $5^{\text {th }}$ IEEE International Symposium on Assembly and Task Planning, 2003, pp. $7-12$.

[22] PI Piezo Ceramic Technology, http://www.piceramic.de/, 2005. 